\title{
A Cross-Sectional Study to Understand 3D Facial Differences in a Population of African Americans and Caucasians
}

\author{
Chung H. Kau ${ }^{1, \odot ~ J u e ~ W a n g ~}{ }^{1}$ Matthew Davis ${ }^{1}$ \\ ${ }^{1}$ Department of Orthodontics, University of Alabama at \\ Birmingham, Birmingham, Alabama, United States
}

\begin{abstract}
Address for correspondence Chung H. Kau, BDS, MScD, MBA, PhD, FDSEdin, FDSGlas, FFDIre, MOrthEdin, FICA, FACD, Department of Orthodontics, University of Alabama, Suite 305, 1919 7th Ave. South, Birmingham, AL 35294, United States (e-mail: ckau@uab.edu).
\end{abstract}

\begin{abstract}
Keywords

- 3D faces

$-3 D$ imaging

- orthodontics

Objective The purpose of this cross-sectional retrospective study was to use three-dimensional surface imaging to determine gender dimorphism and facial morphological changes from adolescence to adulthood in African American and Caucasian populations.

Materials and Methods Three-dimensional images were captured and the total sample size included 371 subjects. Images were combined using Rapidform 2006 Plus Pack 2 software to produce a male and female facial average for each population. Comparisons were conducted within the following categories: (1) gender comparison within each race, (2) adult and adolescent comparison within each race, and (3) adult and adolescent comparison between the races.

Results Adolescent gender comparisons within each race showed high percentages of similarity. However, adult females in both races showed more prominent periorbital, malar, and nasolabial regions and less prominent lower forehead, nose, and lower face compared with adult males of the same race. African American adult females showed increase in length and width of the face, increased nasal tip projection, and decreased periorbital regions compared with African American adolescent females. Welsh adult females had an increase in the nose and chin projection compared with Welsh adolescent females. Adult males of both races had increase in nose and chin projection, increase in length and width of the face, and decreased periorbital, malar, and nasolabial regions compared with adolescent males of the same race. African American adolescents had a wider alar base, more protrusive lips, and periorbital regions, and less prominent nose and chin compared with the Welsh adolescents. African American adults also had a wider alar base; more protrusive lips and periorbital regions; a broader face; and more retrusive chin, nose, nasolabial region; and lower forehead compared with Welsh adults.

Conclusions Few differences were noted between genders within the same racial groups during adolescence. However, changes became more distinct in adulthood. From adolescence to adulthood, facial morphologies were similarly matched within the gender for females; however, there were significant changes for males. Lastly, facial morphology patterns tend to be established early in life.
\end{abstract}




\section{Introduction}

The introduction of three-dimensional (3D) surface imaging technology has expanded researcher's ability to capture and evaluate facial anatomy in its true form. There has been much excitement about what can be further understood about diagnosis, ${ }^{1}$ genetics, ${ }^{2}$ treatment planning, ${ }^{3}$ and treatment execution in orthodontics ${ }^{4}$ and orthognathic surgery. ${ }^{5,6}$

\section{Gender Analysis}

Of interest to many clinicians is the topic of facial differences in gender. Ferrario et $\mathrm{al}^{7}$ evaluated soft tissue facial growth with 3D computerized mesh diagram analysis utilizing wireless markers positioned on selected facial landmarks. They evaluated 1,157 healthy white children and adolescents aged 6 to 17 years and 191 healthy white adults. Average values and a standardized mesh were computed for each age class and gender. Their results showed that males and females had similar growth patterns until age 11 , after which differences were seen. Facial shape differences were most pronounced at the forehead, nose, and chin. Males had larger foreheads, longer noses, and more inferior and prominent lips in every age group. ${ }^{7}$ Another study evaluated gender differences utilizing composite facial templates from a 3D laser scanning system. ${ }^{8}$ Eighty adults with an average age of 24 years and 5 months were scanned. Facial templates were formed for males and females and the results showed the greatest differences were at the nose, zygomatic region, and lower border of the mandible. ${ }^{8}$

In recent times, cross-sectional data from the 3D Facial Norms Database has been used to investigate craniofacial sexual dimorphism in healthy children, adolescents, and adults. 3D facial scans were taken on 1,555 individuals between ages 3 and 25 who were of self-reported European ancestry with no history of craniofacial trauma, congenital malformation, or surgery. ${ }^{9} X, Y$, and $Z$ coordinates corresponding to standard soft tissue landmarks and linear measurements were identified on each scan and 29 anthropometric measurements were analyzed to compare genders. Their results showed significant sex differences at every age group. The magnitude of dimorphism increased with age, showing large differences in the nasal, cranial, and facial measurements after puberty. Significant facial shape differences were also seen at each age group. The authors concluded that several soft tissue sex differences were already present in the youngest age group, indicating that these differences emerged prior to 3 years of age. The authors further noted the complexity of gender dimorphism, as different groups of traits exhibited distinct patterns of dimorphism. The adult male and female facial shapes arose from numerous distinct changes at earlier stages, but were present after puberty. ${ }^{9}$

\section{Population Comparison with Three-Dimensional Surface Imaging}

As the accessibility of 3D surface imaging systems has improved, there has been an increase in the study of facial morphology. Studies comparing facial morphology of different racial groups using various 3D surface imaging technology have increased in the literature. These studies show how different facial morphologies are unique to various ethnicities and present the need to establish facial normal values for population groups around the world. 3D facial averages for five populations including: Wales, Texas, Slovenia, Hungary, and Egypt have been used to describe facial differences. ${ }^{10} \mathrm{~A}$ total of 473 subjects of age 18 to 30 years had 3D surface imaging scans completed. All subjects were of ethnic decent from their native country, had class I malocclusion, no adverse skeletal deviation, normal body mass index (BMI), and no craniofacial anomalies. Average facial shells were constructed for each population and gender and then compared with one another. The authors found there were distinct facial differences among the groups, especially when comparing totally different ethnic variations. ${ }^{10}$

\section{Facial Morphology of Caucasians and African Americans}

Three-dimensional representations of the African American face are not widely reported in the literature. Talbert et al ${ }^{11}$ compared facial morphology of an adult African American population to an adult Caucasian American population using 3D surface imaging. Subjects were 19 to 30 years of age with normal BMI, and no gross craniofacial anomalies. The results showed that African American males had a more prominent upper forehead and periocular region, wider alar base, and more protrusive lips compared with Caucasian males. African American females had a broader face, wider alar base, and more protrusive lips than Caucasian females. Further, Caucasian females showed a more prominent chin point, malar region, and lower forehead compared with African American females. ${ }^{11}$

\section{Aims of the Study}

The purpose of this study was to use 3D surface imaging to determine the following: (1) Facial morphological changes from adolescence to adulthood in African American and Caucasian populations. (2) Facial morphological differences between genders of African American and Caucasian populations.

\section{Materials and Methods}

For this retrospective cross-sectional study, previously collected samples of adolescent and adult subjects of African American and Caucasian descent were selected for evaluation. Information about each group is listed below. The project received approval by the Institutional Review Board UABX2016-Ortho-003. A detailed description can be found in - Table 1.

\section{Imaging Systems}

Two 3D imaging systems were used for image capture of the subjects. The Welsh adolescent and adult groups were captured using a laser-scanning system consisting of two high-resolution Minolta Vivid VI900 3D cameras (Konica Minolta, Osaka, Japan) operating as a stereo pair with 
Table 1 Sample size and the age range

\begin{tabular}{|l|l|l|l|l|}
\hline Populations & Male & Female & Total & Age range $(\mathbf{y})$ \\
\hline AA adolescents (Selma, AL) & 45 & 62 & 107 & $10-13$ \\
\hline AA adults (Birmingham, AL) & 50 & 50 & 100 & $19-30$ \\
\hline Welsh adolescents (Cardiff, UK) & 36 & 28 & 64 & $10-13$ \\
\hline Welsh adults (Cardiff, UK) & 50 & 50 & 100 & $18-30$ \\
\hline
\end{tabular}

Abbreviation: AA, African American.

reported manufacturing accuracy of $0.3 \mathrm{~mm} .{ }^{12}$ The African American adolescent and adult groups were captured using the 3dMDface system (3dMD; Atlanta, Georgia, United States). As reported previously, this system uses a combination of structured light and stereophotogrammetry. Three cameras were positioned on each side of the subject, two infrared and one color. They were synchronized with an industrial grade flash system to simultaneously capture a single image. The system is reported to have an accuracy range of 0.1 to $0.5 \mathrm{~mm} .{ }^{13}$ All subjects sat on an adjustable stool and looked into a mirror. Seat height was adjusted for each subject to achieve natural head position. Subjects were instructed to swallow hard and keep their jaws relaxed just before the scans were taken. Consistency of images from both scanners was evaluated using a phantom head made out of plaster cast. The reproducibility of five separate scans on each machine showed a reproducibility within $0.5 \mathrm{~mm}$ for the representation of the plaster cast.

\section{Processing of Facial Shells}

Once the images were collected for each subgroup, they were transferred to the Rapidform 2006 Plus Pack 2 (RF6 PP2) software (INUS Technology; Seoul, Korea) for analysis. The images were cleaned up by removing distractors such as hair and shoulders so only the subject's facial form was visible. Any surface defects on the images were automatically filled in by the software. After cropping and aligning each face, a facial shell was produced for each subject.

\section{Average Face Construction}

All facial shells in each subgroup were aligned and combined to create an average facial shell for each subgroup. The best fit algorithm from previously validated routines in the RF6 software was used to construct each average shell. This process has been well documented in the literature ${ }^{12}$ and can be summarized by the following steps:

1. Align with best fit using the built-in algorithm in the RF6 software.

2. Average the $z$ coordinates of the images based on normal images to a facial template.

3. Triangulate the point cloud to obtain an average face.

4. Improve the average face by filling in small holes and removing defects.

5. Apply color texture.

6. Create shells with one positive and one negative standard deviation.

\section{Subgroup Comparison}

Eight average facial shells were created from the population subgroups: African American adolescent male (AA-aM), African American adolescent female (AA-aF), African American adult male (AA-AM), African American adult female (AA-AF), Welsh adolescent male (W-aM), Welsh adolescent female (W-aF), Welsh adult male (W-AM), and Welsh adult female (W-AF). To understand and quantify the differences between two shells, a special method of superimposition of shells was performed. Five predetermined anatomical points were identified on each average facial shell: a point at the inner canthus of each eye, a point at each commissure of the lips, and a point at the tip of the nose. The best fit algorithm utilized by the RF6 software was used to superimpose the average shells onto one another for comparison. The average facial shell comparisons discussed in this study are as follows:

1. Female and male comparison within each race.

$\mathrm{A}-\mathrm{aF}$ versus $\mathrm{AA}-\mathrm{aM}, \mathrm{AA}-\mathrm{AF}$ versus $\mathrm{AA}-\mathrm{AM}, \mathrm{W}-\mathrm{aF}$ versus $\mathrm{W}$-aM, W-AF versus $\mathrm{W}$-AM

2. Adult and adolescent comparison within each race.

AA-AM versus AA-aM, AA-AF versus AA-aF, $\mathrm{W}$-AM versus $\mathrm{W}$-aM, $\mathrm{W}-\mathrm{AF}$ versus $\mathrm{W}$-aF

3. African American and Welsh comparison.

$A A-a F$ versus $\mathrm{W}-\mathrm{aF}, \mathrm{AA}-\mathrm{aM}$ versus $\mathrm{W}-\mathrm{aM}$, AA-AF versus W-AF, AA-AM versus $W-A M$

\section{Parameters Measured}

The parameters measured for each subgroup comparison were: linear measurements in millimeters $(\mathrm{mm})$, color histograms, and surface areas and shapes. These parameters have also been previously documented ${ }^{12}$ and are described below.

\section{Linear Measurements}

The software automatically recorded linear measurements to assess differences between the superimposed facial shells. The mean of all the linear measurements was calculated by taking an absolute value of the average difference between the surfaces of two shells. The absolute distance between the two faces was recorded as a positive number rather than providing directional information such as positive or negative. The linear measurements were recorded in millimeters and consist of the maximum and minimum values, average distance, and the standard deviation. The minimum values are always $0 \mathrm{~mm}$ because they are expressed in absolute values of the differences. 


\section{Color Histograms}

Color histograms are maps that compare two facial shells in three dimensions. The color spectrum display allows visualization of the similarities and differences between the two shells. There are two types of color histograms displayed by the RF6 PP2 software in this study: absolute and signed histograms. To discern the information presented in a color histogram, one must know which facial shell is the reference. In this study, the reference shell was always listed as the second shell.

Absolute color histograms simply show gross differences between the two facial shells being compared. Signed color histograms have the differences expressed as vector values, showing either a negative or positive value of one facial shell in relation to the reference shell. ${ }^{4}$ For differences outside the tolerance level, the negative values represent retrusion or a deficient region and are displayed in different shades of blue, while positive values represent protrusion and are displayed in different shades of red. Again, the black color represents variations within the set tolerance level.

\section{Surface Areas and Shapes}

RF6 software automatically creates the surface areas and shapes based on the set tolerance level for the shell comparison. As reported earlier, a study by Kau et $\mathrm{al}^{14}$ showed that $90 \%$ of facial shells were reproducible to within $0.85 \mathrm{~mm}$. Tolerance level for this study was set to $1 \mathrm{~mm}$ to incorporate errors in reproducibility and also in camera configurations.
Any variation between the compared shells under $1 \mathrm{~mm}$, will be considered within acceptable similarity limits and represented by a black color. The areas where the shells deviate more than $1 \mathrm{~mm}$ from each other will show shape and color deviation according to the color spectrum for the absolute and signed histograms.

\section{Results}

The total sample size for this study included 371 subjects (62 AA-aF, 45 AA-aM, 50 AA-AF, 50 AA-AM, $28 \mathrm{~W}$-aF, $36 \mathrm{~W}-\mathrm{aM}, 50 \mathrm{~W}-\mathrm{AF}$, and $50 \mathrm{~W}-\mathrm{AM})$. An average facial shell was constructed for each subgroup: AA-aF, AA-aM, AA-AF, AA-AM, W-aF, W-aM, W-AF, and W-AM. The average facial shells were then superimposed for comparison. A sample of each average face is seen in - Fig. 1 .

\section{Linear Measurements}

- Table 2 is a representation of the absolute linear measurements between the subgroups. The absolute values of the average distance between two shells, the standard deviations, and the maximum and minimum distances are listed for each subgroup comparison. The minimum distance is $0 \mathrm{~mm}$ for all absolute measurements. The largest absolute average distance among the population subgroup comparisons was $1.87 \mathrm{~mm}$ seen between W-AM and $\mathrm{W}$-aM. The smallest absolute distance was $0.42 \mathrm{~mm}$ seen between AA-aF and AA-aM.

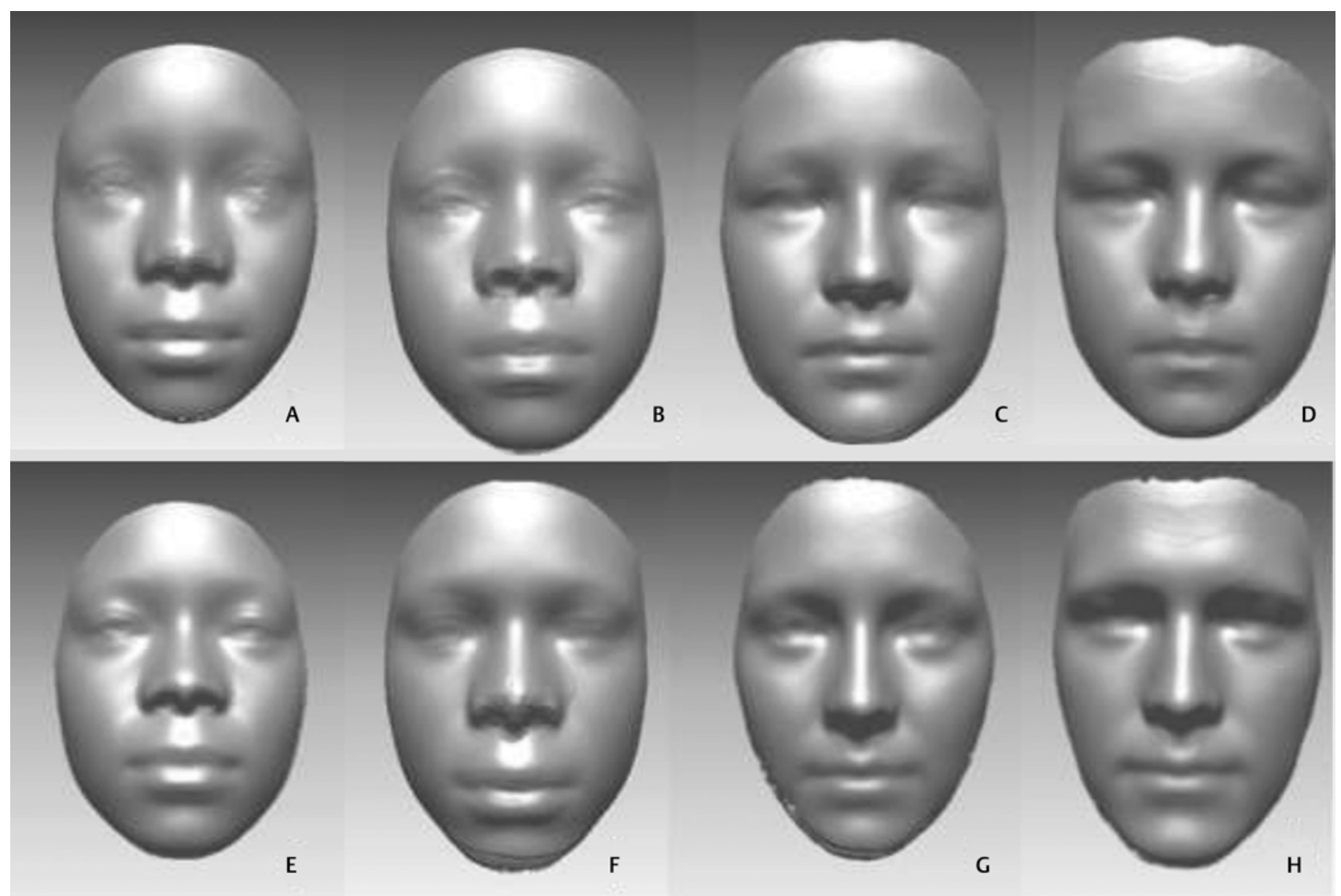

Fig. 1 Average for each face. (A) AA-aF, (B) AA-aM, (C) W-aF, (D) W-aM, (E) AA-AF, (F) AA-AM, (G) W-AF, and (H) W-AM. 
Table 2 Absolute linear measurements showing differences between the facial shells

\begin{tabular}{|l|l|l|l|l|}
\hline Comparison groups & $\begin{array}{l}\text { Average distance } \\
(\mathbf{m m})\end{array}$ & $\begin{array}{l}\text { Standard deviation } \\
(\mathbf{m m})\end{array}$ & $\begin{array}{l}\text { Maximum distance } \\
(\mathbf{m m})\end{array}$ & $\begin{array}{l}\text { Minimum distance } \\
(\mathbf{m m})\end{array}$ \\
\hline AA-aF versus AA-aM & 0.42 & 0.35 & 15.64 & 0.00 \\
\hline AA-AF versus AA-AM & 1.17 & 0.98 & 5.08 & 0.00 \\
\hline W-aF versus W-aM & 0.46 & 0.36 & 2.07 & 0.00 \\
\hline W-AF versus W-AM & 1.29 & 1.04 & 6.04 & 0.00 \\
\hline AA-AF versus AA-aF & 0.90 & 0.79 & 5.92 & 0.00 \\
\hline AA-AM versus AA-aM & 1.72 & 1.32 & 31.90 & 0.00 \\
\hline W-AF versus W-aF & 0.55 & 0.45 & 2.91 & 0.00 \\
\hline W-AM versus W-aM & 1.87 & 1.92 & 12.68 & 0.00 \\
\hline AA-aF versus W-aF & 1.09 & 1.09 & 6.54 & 0.00 \\
\hline AA-aM versus W-aM & 0.95 & 1.03 & 5.81 & 0.00 \\
\hline AA-AF versus W-AF & 1.23 & 1.27 & 6.91 & 0.00 \\
\hline AA-AM versus W-AM & 1.25 & 1.30 & 29.99 & 0.00 \\
\hline
\end{tabular}

Table 3 Signed color map measurements showing differences between the facial shells

\begin{tabular}{|l|l|l|l|l|l|}
\hline Comparison groups & $\begin{array}{l}\text { Average distance } \\
(\mathbf{m m})\end{array}$ & $\begin{array}{l}\text { Standard } \\
\text { deviation }(\mathbf{m m})\end{array}$ & $\begin{array}{l}\text { Maximum } \\
\text { distance }(\mathbf{m m})\end{array}$ & $\begin{array}{l}\text { Minimum } \\
\text { distance }(\mathbf{m m})\end{array}$ & Similarity \\
\hline AA-aF versus AA-aM & -0.067 & 0.55 & 1.96 & -15.65 & 92.85 \\
\hline AA-AF versus AA-AM & 0.182 & 1.52 & 5.08 & -4.05 & 50.51 \\
\hline W-aF versus W-aM & 0.101 & 0.57 & 1.94 & -2.07 & 70.13 \\
\hline W-AF versus W-AM & 0.385 & 1.62 & 3.99 & -6.04 & 49.41 \\
\hline AA-AF versus AA-aF & 0.293 & 1.16 & 5.92 & -2.67 & 84.95 \\
\hline AA-AM versus AA-aM & 0.370 & 2.14 & 7.55 & -31.90 & 36.88 \\
\hline W-AF versus W-aF & 0.080 & 0.71 & 2.91 & -2.13 & 88.55 \\
\hline W-AM versus W-aM & 0.671 & 2.60 & 12.68 & -4.39 & 36.18 \\
\hline AA-aF versus W-aF & 0.232 & 1.53 & 6.54 & -3.98 & 50.71 \\
\hline AA-aM versus W-aM & 0.236 & 1.38 & 6.81 & -4.40 & 68.39 \\
\hline AA-AF versus W-AF & 0.430 & 1.72 & 5.18 & -5.11 & 56.70 \\
\hline AA-AM versus W-AM & 0.098 & 1.80 & -29.99 & 56.65 \\
\hline
\end{tabular}

- Table 3 displays the signed color map measurements for the subgroup comparisons. The average distance, standard deviation, maximum distance, minimum distance, and percent similarity is listed for each comparison. Negative values denote retrusion and positive values denote protrusion of the first facial shell compared with the second (reference) shell. The percent similarity displays the degree of similarity between the two facial shells being compared. The average linear measurements ranged from-0.067 (AA-aF vs. AA-aM) to 0.671 (W-AM vs. W-aM). The highest percent similarity was 92.85\% (AA-aF vs. AA-aM) and the lowest was $36.18 \%$ (W-AM vs. W-aM).

\section{Color Histograms}

As previously discussed, color histograms are maps which use a color spectrum to locate and quantify similarities and differences between two average facial shells. When evaluating the absolute histograms, it is important to remember that the discrepancy between the facial shells increases as the color ranges from blue to red. -Figs. 2-5 show the signed color histograms for all the subgroups. The areas of blue and red correspond respectively to retrusion and protrusion of the first facial shell compared with the second (reference) facial shell in the signed histograms.

\section{Surface Area and Shapes}

The differences of surface area and shapes between the compared facial shells can be visualized with the histograms below. -Figs. 6-12 show the signed histograms for the female and male comparisons for all the race group comparisons.

\section{Discussion}

The purpose of this study was to use 3D imaging to determine gender dimorphism and compare facial 
African

American

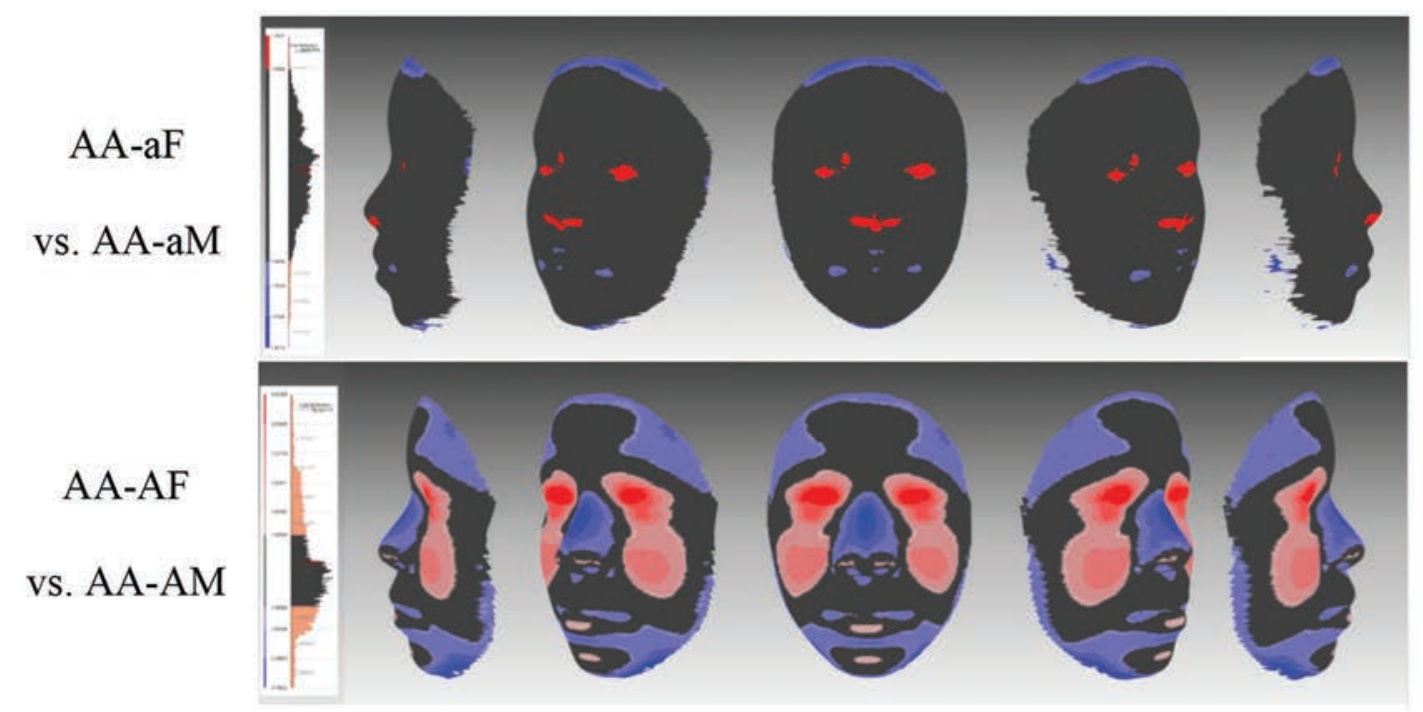

Fig. 2 Signed color histogram for AA-aF versus W-aF.

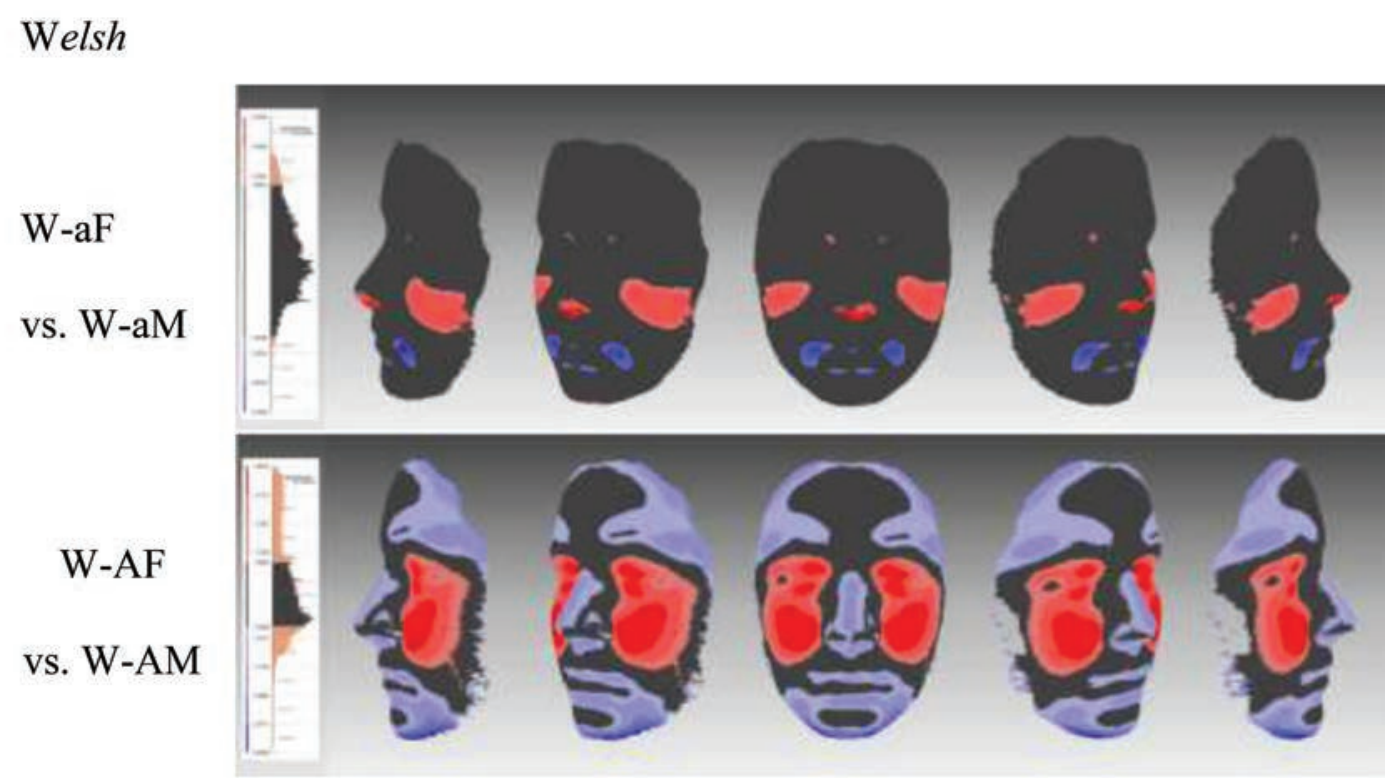

Fig. 3 Composite signed color histograms for female versus male comparison within each race: AA-aF versus AA-aM, AA-AF versus AA-AM, $\mathrm{W}$-aF versus $\mathrm{W}$-aM, and $\mathrm{W}$-AF versus $\mathrm{W}$ - $\mathrm{AM}$. The areas of blue and red correspond respectively to retrusion and protrusion of the first facial shell compared with the second (reference) facial shell.

morphological differences from adolescence to adulthood in African American and Caucasian populations. The average facial shells of the subgroups were compared to determine the differences within the following categories: (1) female and male comparison within each race, (2) adult and adolescent comparison within each race, and (3) adult and adolescent comparisons between the races. The color histogram results for these categories showed significant differences and are discussed below.

\section{Female/Male Comparison within Each Race}

The adolescent female and male comparisons for each race showed high percentages of similarity at $92.85 \%$ (AA-aF vs. AA-aM) and $90.13 \%$ (W-aF vs. W-aM). This indicates that female and male adolescents of the same race have similar facial traits and forms from 10 to 13 years of age. Although there was a high percentage of similarity, the average African American adolescent female face showed some prominence at the eyes, alar base, and columella, while 


\section{African American}
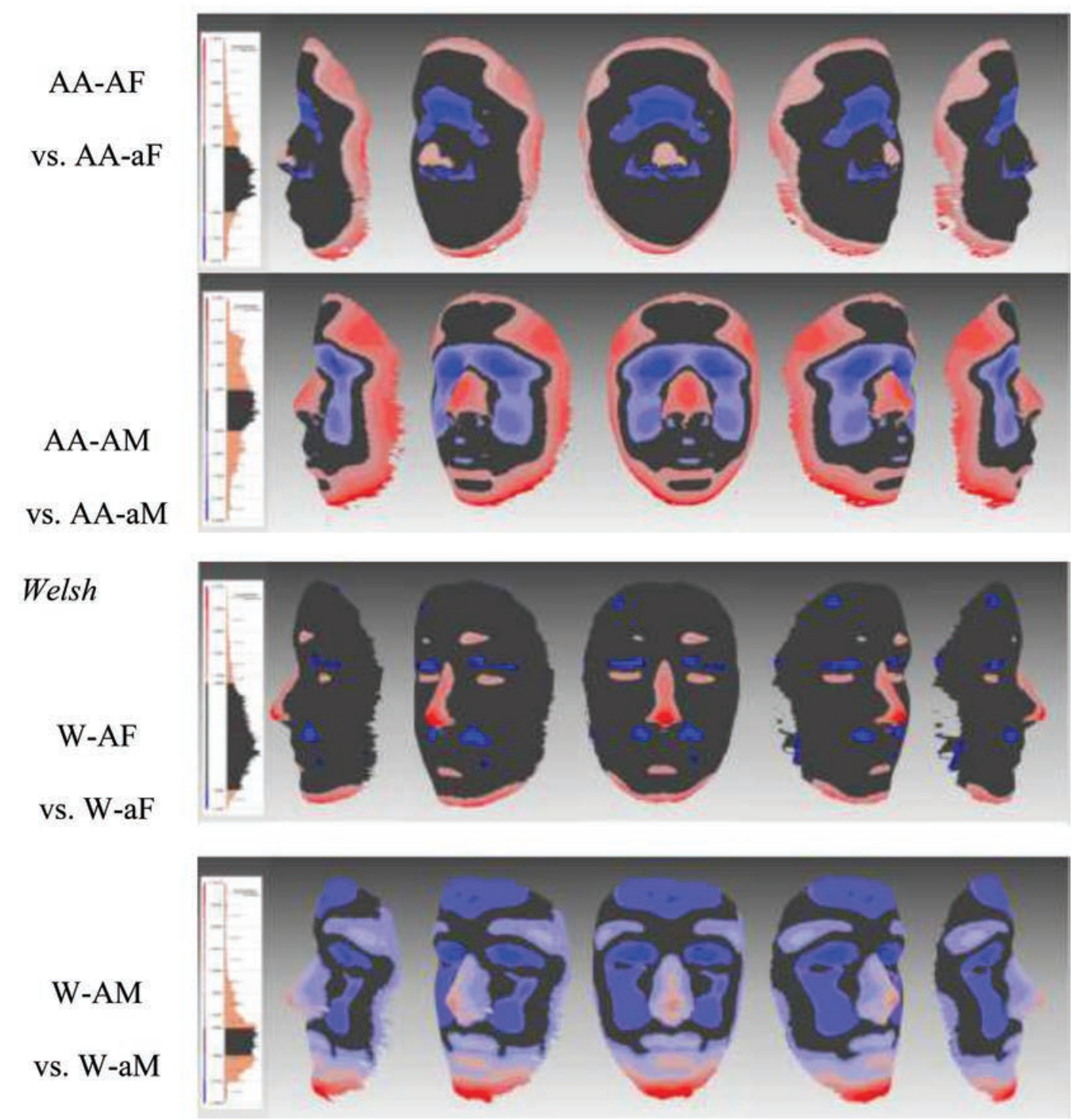

Fig. 4 Composite signed color histograms for adult versus adolescent comparison within each race: AA-AF versus AA-aF, AA-AM versus AA-aM, $\mathrm{W}$-AF versus $\mathrm{W}$-aF, and $\mathrm{W}$-AM versus $\mathrm{W}$-aM. The areas of blue and red correspond respectively to retrusion and protrusion of the first facial shell compared with the second (reference) facial shell.

exhibiting retrusion at the commissures compared with the average African American adolescent male. Similarly, the average Welsh adolescent female face had more prominent malar regions, alar base, and columella, while showing less prominent commissural areas compared with the Welsh adolescent male. The adult female and male comparisons for each race showed less similarity than during the adolescent time period; however, the differences followed a similar pattern in both races. The AA-AF versus AA-AM were $50.51 \%$ similar and the $\mathrm{W}$-AF versus $\mathrm{W}$-AM were $49.41 \%$ similar. The African American and Welsh adult females showed more prominent periorbital, malar, submalar, and nasolabial fold regions and more retrusive supraorbital, nasal, labiomental, and soft tissue menton regions compared with the African American and Welsh adult males. The decrease in similarity of the adult subgroups compared with the adolescent subgroups signifies that facial traits change with growth and may be specific to gender.

\section{Adult/Adolescent Comparison within Each Race}

Corresponding adult and adolescent subgroups were compared with distinguish areas of facial change from adolescence to adulthood according to gender and race. African American females (AA-AF vs. AA-aF) had $84.95 \%$ similarity from adolescence to adulthood. Increases in nasal projection, length, and width of the face were noted as well as retrusion of the periorbital, glabellar, soft tissue nasion, and nasolabial fold areas. At $36.88 \%$ similarity, the African American adult males (AA-AM vs. AA-aM) demonstrated 


\section{Adolescents}

AA-aF

vs. W-aF
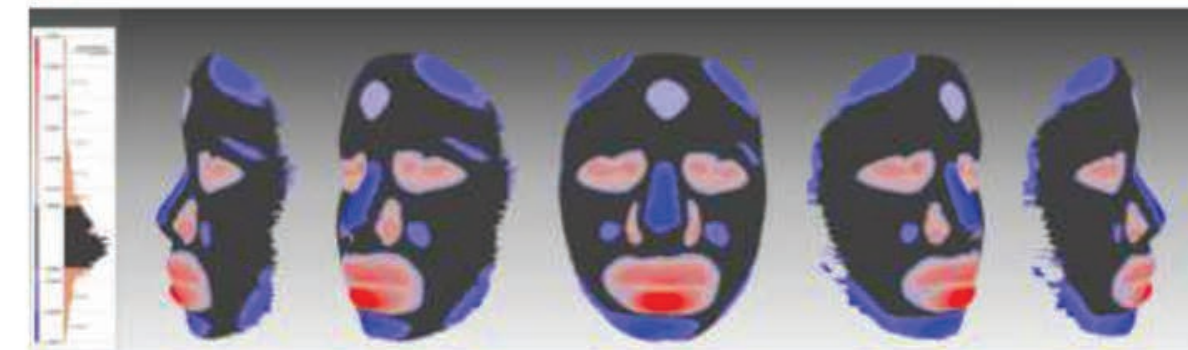

AA-aM

vs. W-aM
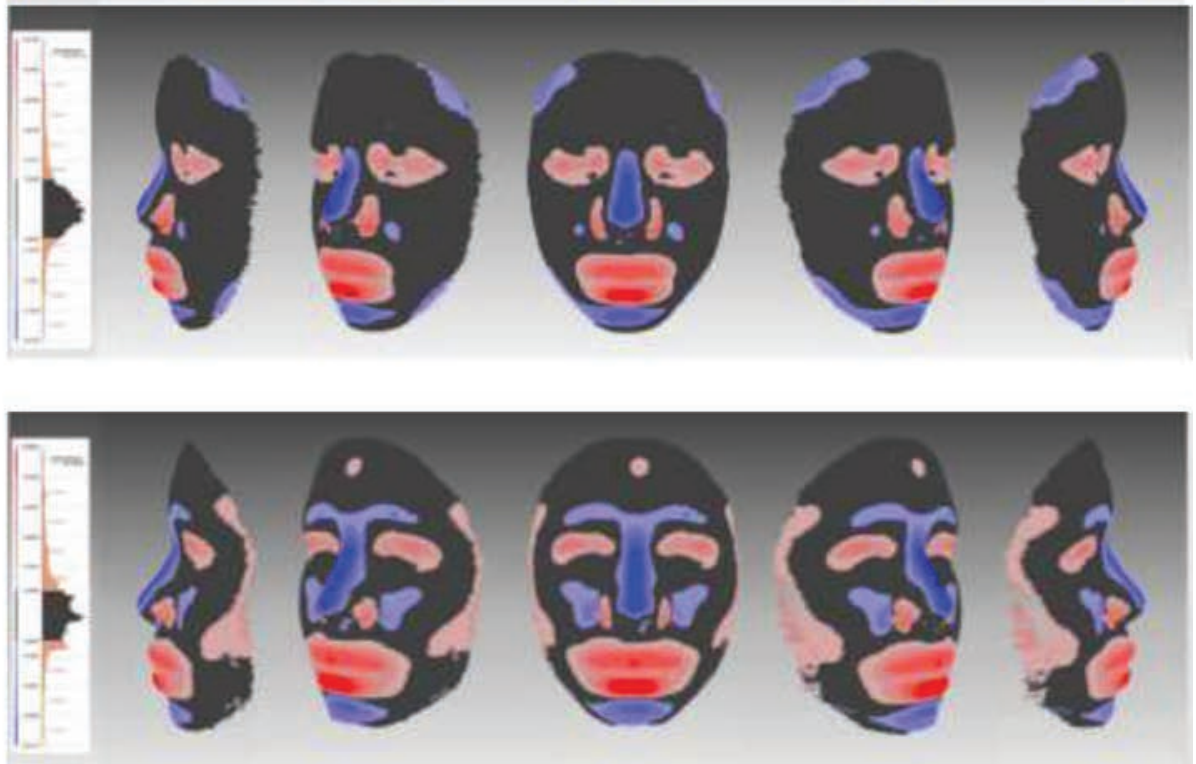

Adults

$$
\text { AA-AF }
$$

vs. W-AF

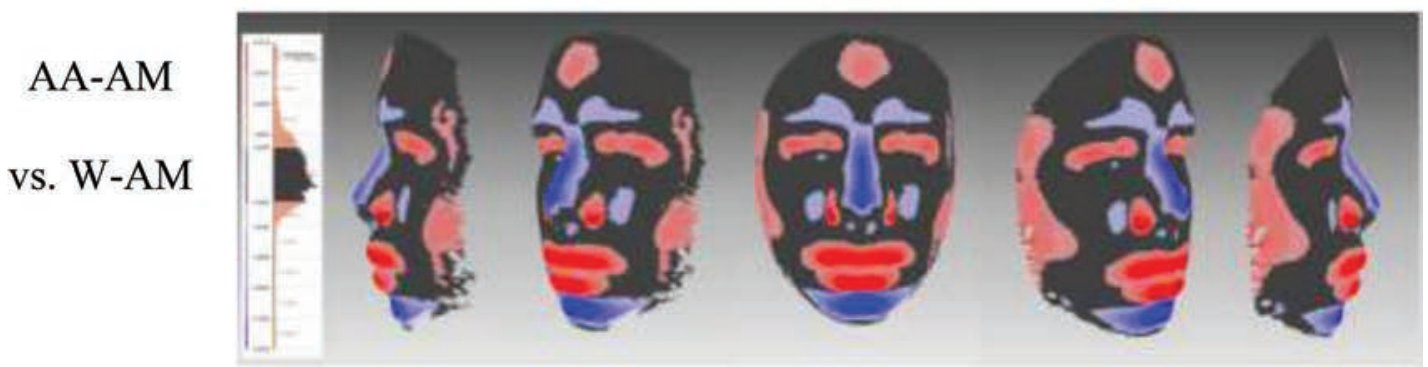

Fig. 5 Composite signed color histograms for subgroups: $A A-a F$ versus $W$-aF, AA-aM versus $W$-aM, AA-AF versus $W-A F$, and $A A-A M$ versus $\mathrm{W}$-AM. The areas of blue and red correspond respectively to retrusion and protrusion of the first facial shell compared with the second (reference) facial shell.

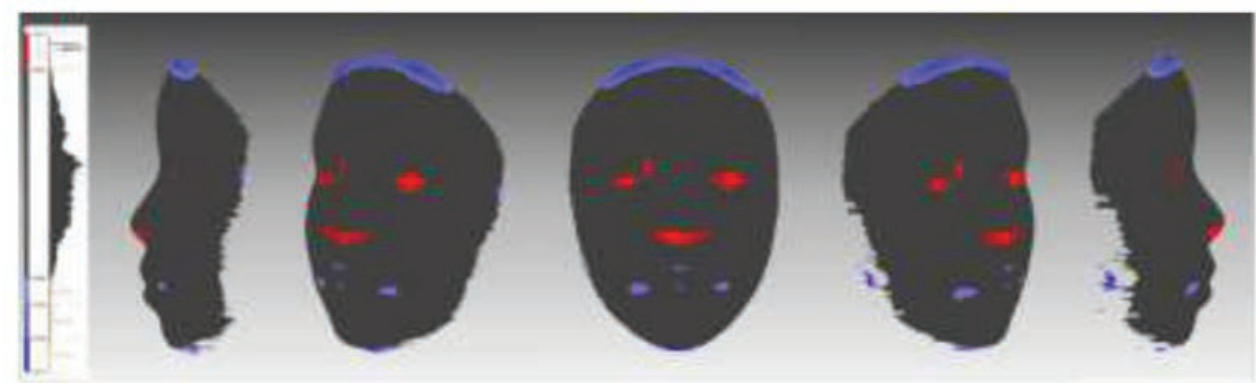

Fig. 6 AA-aF versus AA-aM signed histogram comparison. The average faces for African American adolescent males and females showed high similarity at $92.85 \%$. The average African American adolescent female face showed slight prominence at the eyes, an increased alar base, and columella, while showing retrusion at the commissures compared with the average African American adolescent male. 


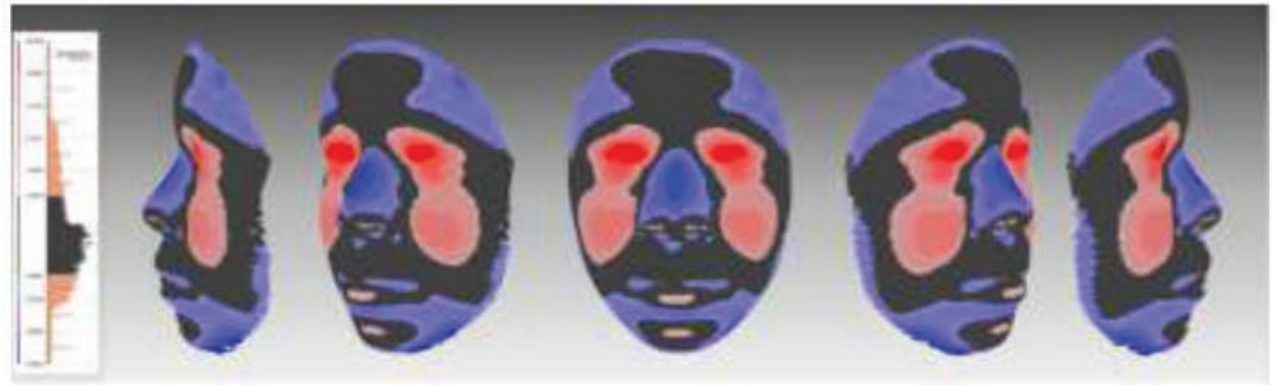

Fig. 7 AA-AF versus AA-AM signed histogram comparison. The African American adult females showed more prominent periorbital, malar, and submalar regions and retrusive nasal dorsum, labiomental region, commissures, and cranial width compared with the African American adult males.

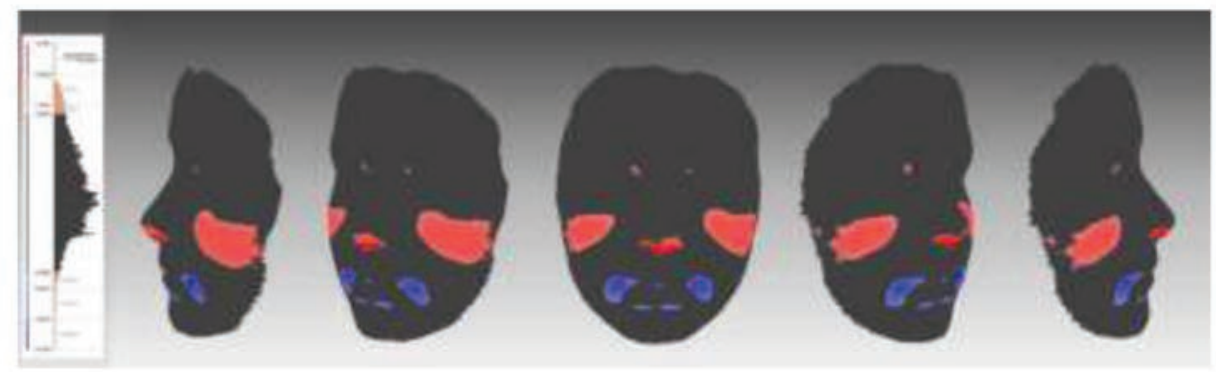

Fig. $8 \mathrm{~W}$-aF versus W-aM signed histogram comparison. The average Welsh adolescent female face had more prominent malar regions, wider alar base, and columella, while showing less prominent commissural areas compared with the Welsh adolescent male.

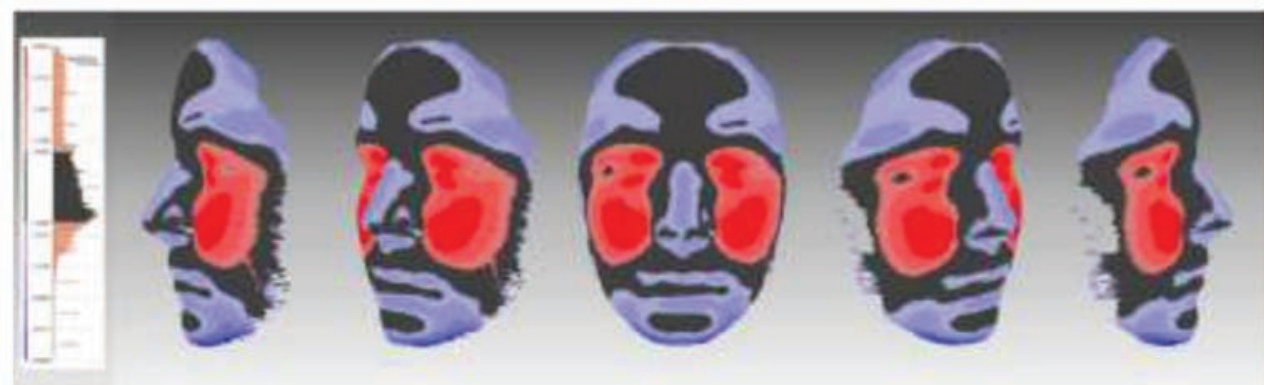

Fig. 9 W-AF versus W-AM signed color histogram comparison. Similar to the African American adult female and male comparison, the Welsh females had more prominent periorbital, malar, submalar, and nasolabial fold regions and more retrusive nasal dorsum, columella, alar base, labiomental region, soft tissue menton, and cranial width compared with the males.

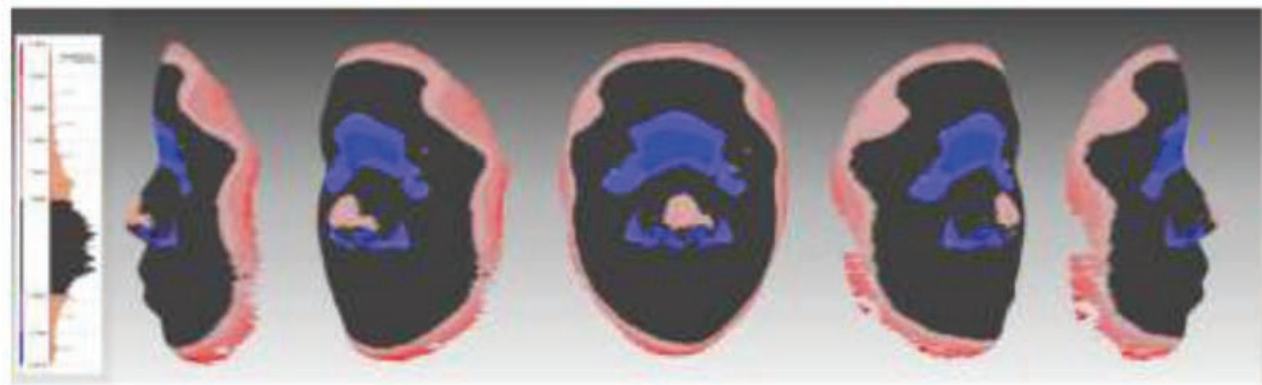

Fig. 10 AA-AF versus AA-aF signed color histogram comparison. The African American adult female had increased prominence at the nasal tip area and showed an overall increase in width and length of the face. However, the adult also showed areas of retrusion at the periorbital, soft tissue nasion, glabella, and nasolabial fold areas in comparison to the adolescent female.

much less similarity to the adolescent males as compared with their female counterparts. The adult male showed an increased prominence of the nose and overall increase in length and width of the face, as well as retrusion of the periorbital, malar, submalar, and nasolabial areas compared with the adolescent male. However, generally speaking, the adult African American male showed similar yet more pronounced changes from adolescence compared with that of African American females.

The comparison of Welsh adult and adolescent females (W-AF vs. W-aF) also generated a high level of similarity, at $88.55 \%$. The adult female displayed a prominent nasal 


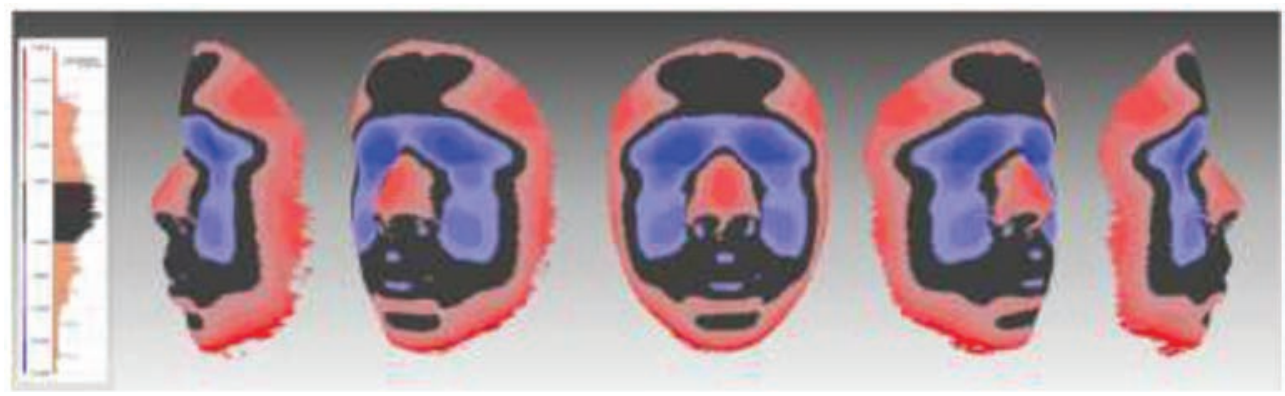

Fig. 11 AA-AM versus AA-aM signed color histogram comparison. The African American adult male showed increased nasal projection and an overall increase in length and width. However, the adult also showed retrusion of the periorbital, malar, submalar, and nasolabial regions compared with the adolescent male.

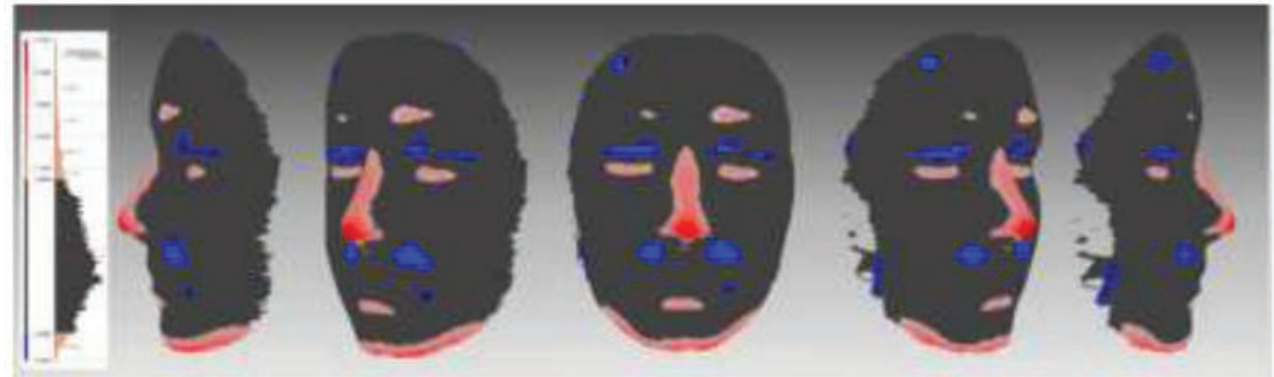

Fig. 12 W-AF versus W-aF signed color histogram comparison. The adult displayed a prominent nasal tip, nasal dorsum, labiomental fold, chin, and soft tissue menton. The adult also displayed some retrusion of the nasolabial fold area compared with the adolescent female.

tip, nasal dorsum, labiomental fold, and soft tissue menton, while showing some retrusion of the nasolabial fold area compared with the adolescent female. The Welsh adult male displayed $36.18 \%$ similarity to the Welsh adolescent male (W-AM vs. W-aM). The adult had an increase in nasal, lip, and chin projection along with a more prominent supraorbital region, but also showed retrusion at the periorbital, malar, submalar, and nasolabial areas when compared with the adolescent male.

When evaluating the changes from adolescence to adulthood in each gender, it is important to note that there was more facial change from adolescence to adulthood in the male groups compared with the female groups. The percent similarities for the female comparisons were 84.95 and $88.55 \%$, compared with 36.88 and $36.18 \%$ for the male comparisons. This could be indicative of pubertal growth occurring earlier in females than males ${ }^{15}$ which would explain how more growth was captured within this data for the males than the females. The smaller amount of change recorded from adolescence to adulthood in both female ethnicities suggests that growth changes could have occurred earlier than when the data was collected.

\section{African American/Welsh Comparison}

The comparison of adolescent and adult female and male subgroups between African American and Welsh races showed similar trends between all comparisons. Both male and female adolescent comparisons (AA-aF vs. W-aF and AA-aM vs. W-aM) displayed more protrusive lips, nasolabial fold, and periorbital regions, and less prominent nose and chin in the African Americans compared with the Welsh. The adult comparisons (AA-AF vs. W-AF and AA-AM vs. W-AM) essentially showed differences in the same locations, but were displayed more prominently compared with the adolescent comparisons, with the addition of broader faces and retrusion being evident at the submalar and glabellar regions of the African American adults compared with the Welsh adults. These findings were very similar to the findings of previous studies of facial analysis of bimaxillary protrusiveness of the African American populations. ${ }^{16,17}$ The subgroup comparisons and respective percent similarities were as follows: AA-aF versus $\mathrm{W}-\mathrm{aF}$ (50.71\%), AA-aM versus $\mathrm{W}$-aM (68.39\%), AA-AF versus $\mathrm{W}-\mathrm{AF}$ (56.70\%), and AA-AM versus W-AM (56.65\%).

\section{Clinical Significance}

This study represents the first time 3D surface imaging has been used to understand population changes and the facial changes that occur cross-sectionally from adolescence to adulthood. Three main findings were noted. First, there were few differences between genders within the same racial groups during the adolescent phase of life. This, however, became more distinct in adulthood (-Fig. 13). Second, facial morphologies were similarly matched within the gender for females but there were significant changes for the males. These findings could be explained by the pubertal spurts happening at different rates for males and females. Since adolescent males have a tendency for facial changes from 12 to 14 years of age, ${ }^{4}$ it is easy to understand the facial changes seen in the age groups (-Fig. 14). Finally, the facial morphology tends to be established early in life. The bimaxillary protrusion, flatness of the nose, and reduced chin projection were evident from the adolescent age groups. These surface shapes changed very little over time and the patterns of differences were maintained throughout the study age groups ( - Fig. 15). 


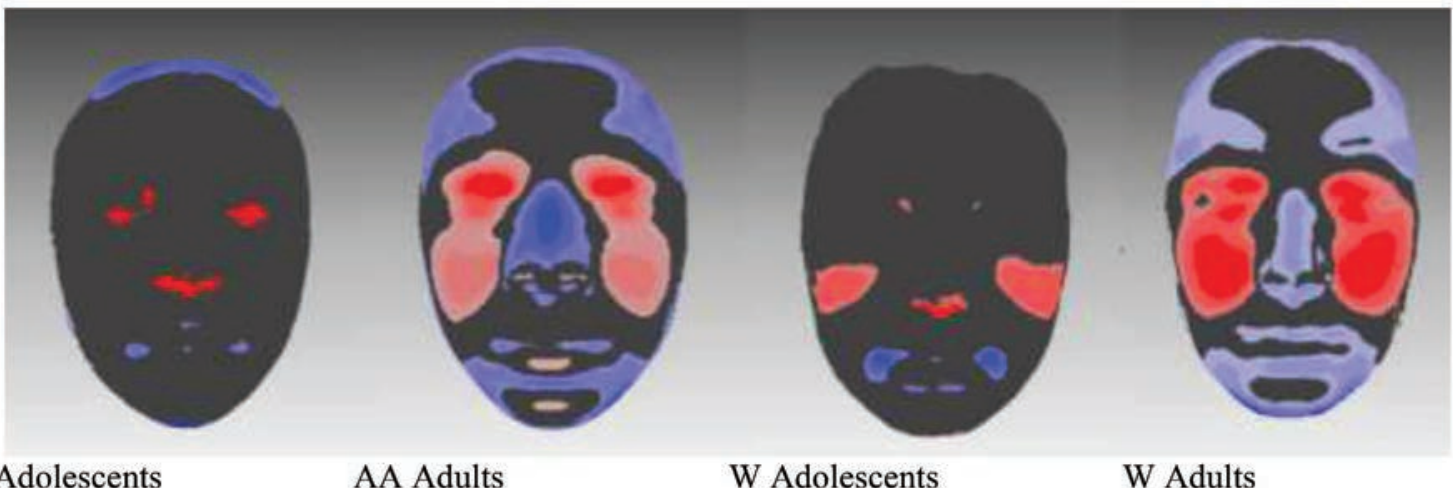

AA Adolescents

AA Adults

W Adolescents

W Adults

Fig. 13 Differences between genders within the same racial groups during the adolescent and adult phases. From left to right: AA-aF versus AA-aM, AA-AF versus AA-AM, W-aF versus $W$-aM, W-AF versus $W$-AM.

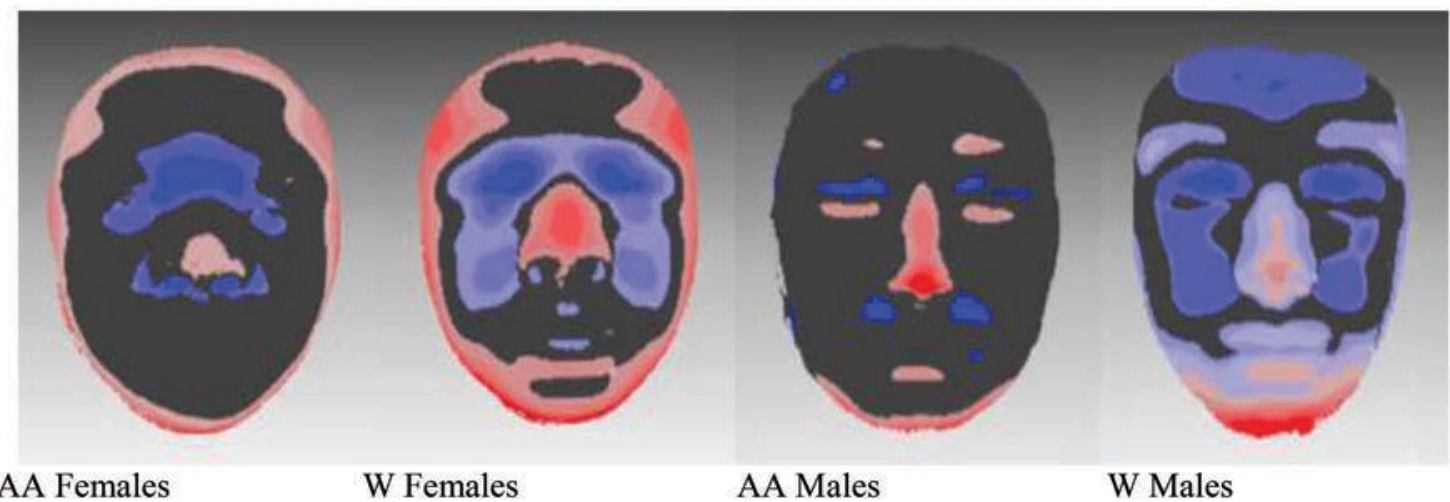

Fig. 14 From adolescence to adulthood, facial morphologies were similarly matched within the gender for females but there were significant changes for the males. From left to right: AA-AF versus AA-aF, AA-AM versus AA-aM, W-AF versus W-aF, W-AM versus W-aM.

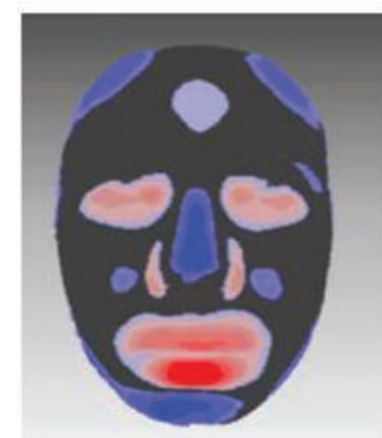

Adolescent

Females

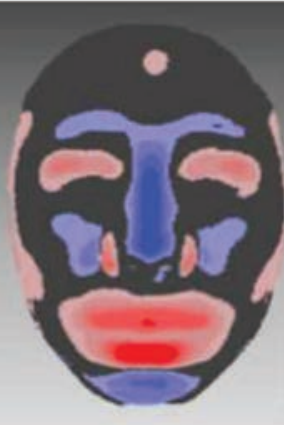

Adult

Females

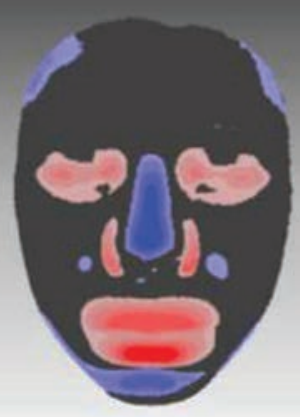

Adolescent

Males

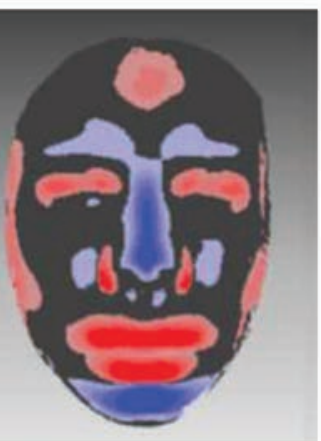

Adult

Males

Fig. 15 Differences between races during the adolescent and adult phases. From left to right: AA-aF versus W-aF, AA-AF versus W-AF, AA-aM versus $\mathrm{W}$-aM, AA-AM versus $\mathrm{W}$-AM.

\section{Limitations to the Study}

There were some limitations to this study. First, this study presents qualitative data in the form of visualization of surface differences of 3D average faces. While the study lacked quantitative outcomes, the ability to visualize the 3D shape and surface area differences were very meaningful to understanding 3D faces at different time points (e.g., adolescent and adulthood). Second, no information on social environment and geographic locations were recorded and these could make the outcome different to the presented results.

\section{Conclusions}

- Few differences were noted between genders within the same racial groups during adolescence. However, changes became more distinct in adulthood.

- From adolescence to adulthood, facial morphologies were similarly matched within the gender for females but there were significant changes for males.

- Facial morphology patterns tend to be established early in life. 
Ethical Approval and Consent to Participate

Ethics approval and consent to participate was obtained from UAB Institution Review Board.

\section{Consent for Publication}

Consent for publication has been obtained by authors and participants as outlined in the IRB.

\section{Availability of Supporting Data}

Supporting data are available on request.

\section{Authors' Contributions}

Dr. Kau was the principal investigator and collected the data, performed the IRB, and prepared the manuscript. Dr. Wang prepared the manuscript and figures. Dr. Davis performed the analysis, wrote the paper, and analyzed the data.

\section{Funding}

None.

\section{Conflict of Interest}

None declared.

\section{References}

1 Weinberg SM. 3D stereophotogrammetry versus traditional craniofacial anthropometry: comparing measurements from the 3D facial norms database to Farkas's North American norms. Am J Orthod Dentofacial Orthop 2019;155(5):693-701

2 Richmond S, Howe LJ, Lewis S, Stergiakouli E, Zhurov A. Facial genetics: a brief overview. Front Genet 2018;9:462

3 Weinberg SM, Cornell R, Leslie EJ. Craniofacial genetics: where have we been and where are we going? PLoS Genet 2018;14(6):e1007438

4 Kau CH, Richmond S. Three-dimensional analysis of facial morphology surface changes in untreated children from 12 to 14 years of age. Am J Orthod Dentofacial Orthop 2008;134(6):751-760
5 Wong ME, Kau CH, Melville JC, Patel T, Spagnoli DB. Bone reconstruction planning using computer technology for surgical management of severe maxillomandibular atrophy. Oral Maxillofac Surg Clin North Am 2019;31(3):457-472

$6 \mathrm{Kau} \mathrm{CH}$, Cronin AJ, Richmond S. A three-dimensional evaluation of postoperative swelling following orthognathic surgery at 6 months. Plast Reconstr Surg 2007;119(7):2192-2199

7 Ferrario VF, Sforza C, Serrao G, Colombo A, Ciusa V. Soft tissue facial growth and development as assessed by the three-dimensional computerized mesh diagram analysis. Am J Orthod Dentofacial Orthop 1999;116(2):215-228

8 Kau CH, Zhurov A, Richmond S, Cronin A, Savio C, Mallorie C. Facial templates: a new perspective in three dimensions. Orthod Craniofac Res 2006;9(1):10-17

9 Kesterke MJ, Raffensperger ZD, Heike CL, et al. Using the 3D Facial Norms Database to investigate craniofacial sexual dimorphism in healthy children, adolescents, and adults. Biol Sex Differ 2016;7:23

10 Kau CH, Richmond S, Zhurov A, et al. Use of 3-dimensional surface acquisition to study facial morphology in 5 populations. Am J Orthod Dentofacial Orthop 2010;137(4 Suppl): S56 e51-59; discussion S56-57

11 Talbert L, Kau CH, Christou T, Vlachos C, Souccar N. A 3D analysis of Caucasian and African American facial morphologies in a US population. J Orthod 2014;41(1):19-29

$12 \mathrm{Kau} \mathrm{CH}$, Zhurov A, Richmond S, et al. The 3-dimensional construction of the average 11-year-old child face: a clinical evaluation and application. J Oral Maxillofac Surg 2006;64(7):1086-1092

13 Lübbers HT, Medinger L, Kruse A, Grätz KW, Matthews F. Precision and accuracy of the 3dMD photogrammetric system in craniomaxillofacial application. J Craniofac Surg 2010;21(3):763-767

14 Kau CH, Richmond S, Zhurov AI, et al. Reliability of measuring facial morphology with a 3-dimensional laser scanning system. Am J Orthod Dentofacial Orthop 2005;128(4):424-430

15 Proffit WR, Fields HW, Sarver DM. Contemporary Orthodontics. 4th ed. St. Louis, MO: Mosby Elsevier; 2007

16 Sushner NI. A photographic study of the soft-tissue profile of the Negro population. Am J Orthod 1977;72(4):373-385

17 Taylor WH, Hitchcock HP. The Alabama analysis. Am J Orthod 1966;52(4):245-265 